\title{
QUANTUM GROUPS AND DOUBLE QUIVER ALGEBRAS
}

\author{
Hua-Lin Huang \\ Mathematics Section, The Abdus Salam International Centre for Theoretical Physics \\ Strada Costiera 11, 34100 Trieste, Italy \\ and \\ Department of Mathematics, University of Science and Technology of China, \\ Hefei 230026, Anhui, P. R. China \\ hualin@ustc.edu.cn \\ Shilin Yang* \\ College of Applied Sciences, Beijing University of Technology \\ Beijing 100022, P. R. China \\ slyang@bjut.edu.cn
}

\begin{abstract}
For a finite dimensional semisimple Lie algebra $\mathfrak{g}$ and a root $q$ of unity in a field $k$, we associate to these data a double quiver $\overline{\mathcal{Q}}$. It is shown that a restricted version of the quantized enveloping algebras $U_{q}(\mathfrak{g})$ is a quotient of the double quiver algebra $k \overline{\mathcal{Q}}$.
\end{abstract}

\section{Introduction}

Let $U_{q}(\mathfrak{g})$ be the Drinfeld-Jimbo quantum group, which is a deformation of the universal enveloping algebra of a finite dimensional semisimple Lie algebra $\mathfrak{g}$. In the generic case, i.e. the parameter $q$ is not a root of unity, several models have been raised to realize it. For example, the Ringel-Hall algebra approach is one successful model among them, see $[10,5,11]$. The case where $q$ is a root of unity is of an particular interest since it is related with the modular representation theory. It is remarkable that a finite dimensional Hopf algebra, so-called restricted version of $U_{q}(\mathfrak{g})$ arose naturally when Lusztig considered this class of quantum

${ }^{*}$ The author is partially supported by the National Science Foundation of China (Grant. 10271014) and Natural Science Foundation of Beijing City(Grant. 1042001)

2000 Mathematics Subject Classification. 81R50, 16W30. 
group at roots of unity. See $[7,8,9]$. Just as the generic case, it is interesting to find an algebra method to realize $U_{q}(\mathfrak{g})$, or its restricted version. In this sense, Cibils $[1,2]$ found that some quotient of a particular path algebra can realize the positive part of the restricted quantized enveloping algebra corresponding to $\mathfrak{g}$. Gordon in [6] extended several results in [2]. We remark that the restricted form of $U_{q}\left(\mathfrak{s l}_{2}\right)$ is realized in the paper [12] by applying the deformation of preprojective algebra introduced in [3].

Let $k$ be a field, $\ell=n$ if $n$ is odd and $\ell=n / 2$ if $n$ is even, where $n \geq 5$. Let $\mathfrak{g}$ be a finite dimensional semisimple simple laced Lie algebra and $q$ an $n$-th primitive root of unity. By $\mathcal{C}$ we denote the associated Cartan matrix of $\mathfrak{g}$. For the Cartan matrix $\mathcal{C}=\left(a_{i j}\right)_{t \times t}$, there is an associated quantum algebra $\mathfrak{u}_{q}(\mathcal{C})$, which by definition, is an associative $k$-algebra with generators $K_{i}, E_{i}, F_{i}$ for $1 \leq i \leq t$, subjecting to the relations

$$
\begin{aligned}
& K_{i}^{n}=1, K_{i} K_{j}=K_{j} K_{i} ; \\
& K_{i} E_{j} K_{i}^{-1}=q^{a_{i j}} E_{j}, K_{i} F_{j} K_{i}^{-1}=q^{-a_{i j}} F_{j} ; \\
& E_{i} F_{j}-F_{j} E_{i}=\delta_{i j} \frac{K_{i}-K_{i}^{-1}}{q-q^{-1}} ; \\
& E_{i}^{\ell}=0, F_{i}^{\ell}=0 ; \\
& \sum_{s=0}^{1-a_{i j}}(-1)^{s}\left[\begin{array}{c}
1-a_{i j} \\
s
\end{array}\right]_{q} E_{i}^{1-a_{i j}-s} E_{j} E_{i}^{s}=0, \text { if } i \neq j ; \\
& \sum_{s=0}^{1-a_{i j}}(-1)^{s}\left[\begin{array}{c}
1-a_{i j} \\
s
\end{array}\right]_{q} F_{i}^{1-a_{i j}-s} F_{j} F_{i}^{s}=0, \text { if } i \neq j,
\end{aligned}
$$

where $[m]_{x}=\frac{x^{m}-x^{-m}}{x-x^{-1}},[m] !_{x}=[m]_{x} \cdots[1]_{x},[0]_{x} !=1$, and $\left[\begin{array}{c}m \\ s\end{array}\right]_{x}=\frac{[m] !_{x}}{[s] !_{x}[m-s] !_{x}}$ for an indeterminate $x$. The algebra $\mathfrak{u}_{q}(\mathcal{C})$ is a Hopf algebra, where the comultiplication, counit, and antipode are given as follows

$$
\begin{aligned}
\Delta\left(K_{i}\right) & =K_{i} \otimes K_{i}, \\
\Delta\left(E_{i}\right) & =E_{i} \otimes 1+K_{i} \otimes E_{i}, \\
\Delta\left(F_{i}\right) & =F_{i} \otimes K_{i}^{-1}+1 \otimes F_{i}, \\
\varepsilon\left(K_{i}\right) & =1, \varepsilon\left(E_{i}\right)=\varepsilon\left(F_{i}\right)=0, \\
S\left(K_{i}\right) & =K_{i}^{-1}, S\left(E_{i}\right)=-K_{i}^{-1} E_{i}, S\left(F_{i}\right)=-F_{i} K_{i} .
\end{aligned}
$$

It would be noted that the restricted quantum group in the sense of Luzstig is some quotient of $\mathfrak{u}_{q}(\mathcal{C})[8,6]$.

As a continuation of [12], the aim of this paper is to construct the quantum group $\mathfrak{u}_{q}(\mathcal{C})$ from a quiver up to Hopf algebra isomorphism for an arbitrary finite dimensional semisimple simply laced Lie algebra $\mathfrak{g}$. Accordingly, we first define a double quiver $\overline{\mathcal{Q}}$ associated to $\mathcal{C}$ 
and $q$, and we get the double quiver algebra $k \overline{\mathcal{Q}}$. Then we describe an algebra $\Pi^{\mathcal{C}}$ obtained from the algebra $k \overline{\mathcal{Q}}$, which is actually a Hopf algebra. Finally, we construct a quotient $\mathfrak{u}_{q}^{\mathcal{C}}$ of $\Pi^{\mathcal{C}}$, which inherits the Hopf algebra structure. We will show that $\mathfrak{u}_{q}(\mathcal{C})$ is isomorphic to $\mathfrak{u}_{q}^{\mathcal{C}}$ as Hopf algebras. It is mentioned that the results in [2] are extended. If $\mathfrak{g}$ is the three dimensional simple Lie algebra, $\Pi^{\mathcal{C}}$ is just a deformation of preprojective algebra in [3] and $\mathfrak{u}_{q}^{\mathcal{C}}$ is the algebra $\mathfrak{u}_{q}$ described in [12].

\section{Preliminaries}

In this section, we list some notations for the convenience of the statement.

Let $k$ be a fixed field. Let us fix an integer $n \geq 5$, an $n$-th primitive root $q$ of unity and a positive definite symmetric Cartan matrix $\mathcal{C}=\left(a_{i j}\right)_{t \times t}$. By $c^{i}$ we denote the $i$-th column of $\mathcal{C}$, by $\mathbb{Z}_{s}$ the cyclic group $\frac{\mathbb{Z}}{s \mathbb{Z}}$ for any positive integer $s$, and by $\underline{t}$ the set $\{1,2, \cdots, t\}$.

For $\alpha=\left(a_{1}, \cdots, a_{t}\right), \beta=\left(b_{1}, \cdots, b_{t}\right) \in \mathbb{Z}_{n}^{t}$, we denote $\alpha \cdot \beta=a_{1} b_{1}+\cdots+a_{t} b_{t}$. The following lemma will be used later on.

Lemma 1.1 If $0 \neq \beta \in \mathbb{Z}_{n}^{t}$, then $\sum_{\alpha \in \mathbb{Z}_{n}^{t}} q^{\alpha \cdot \beta}=0$.

Proof. Obviously, if $n \nmid j$, then $\sum_{i=0}^{n-1} q^{i j}=0$ since $q$ is an $n$-th root of 1 . In general, if $\beta=\left(b_{1}, b_{2}, \cdots, b_{t}\right) \neq 0$, then there exists $i$ such that $b_{i} \neq 0$. We have

$$
\sum_{\alpha \in \mathbb{Z}_{n}^{t}} q^{\alpha \cdot \beta}=\left(\sum_{a_{1} \in \mathbb{Z}_{n}} q^{a_{1} b_{1}}\right) \cdots\left(\sum_{a_{i} \in \mathbb{Z}_{n}} q^{a_{i} b_{i}}\right) \cdots\left(\sum_{a_{t} \in \mathbb{Z}_{n}} q^{a_{t} b_{t}}\right)=0 .
$$

The lemma follows.

Given a positive integer $m$ and a variable $x$, we denote

$$
(m)_{x}=1+x+x^{2}+\cdots+x^{m-1},
$$

and

$$
(m) !_{x}=(m)_{x}(m-1)_{x} \cdots(1)_{x}
$$

We set $(0) !_{x}=1,\left(\begin{array}{c}m \\ u\end{array}\right)_{x}=\frac{(m) !_{x}}{(m-u) !_{x}(u) !_{x}}$ if $u \leq m$ are positive integers. The notations $[m]_{x},[0] !_{x},[m] !_{x}$, and $\left[\begin{array}{c}m \\ s\end{array}\right]_{x}$ are as stated before. 
A quiver $Q$ is an oriented graph given by two sets $Q_{0}$ and $Q_{1}$ of vertices and arrows and two maps $s, t: Q_{1} \rightarrow Q_{0}$ providing each arrow with its source and terminal vertex. A path $\gamma$ is a finite sequence of concatenated arrows $\gamma=a_{n} \cdots a_{1}$, which means that $t\left(a_{i}\right)=s\left(a_{i+1}\right)$ for $i=1, \cdots, n-1$. We set $s(\gamma)=s\left(a_{1}\right)$ and $t(\gamma)=t\left(a_{n}\right)$. It is noted that a vertex $u$ coincides with its source and terminal vertex. The length of a path means the length of its arrow sequence; vertices mean zero-length paths.

The path algebra of $Q$ is a $k$-vector space $k Q$ with the basis $\{p \mid p$ is a path of $Q\}$, where the multiplication of two paths $p$ and $q$ is defined by

$$
p \cdot q= \begin{cases}p q, & \text { if } s(p)=t(q), \\ 0, & \text { otherwise. }\end{cases}
$$

Let $\bar{Q}$ be the double quiver of $Q$. Namely, $\bar{Q}$ is obtained by adding a reverse arrow $a^{*}: j \rightarrow i$ for each arrow $a: i \rightarrow j$ in $Q$. For each vertex $i$, let $e_{i}$ be the associated trivial path.

We always assume that $\ell=n$ if $n$ is odd and $\ell=n / 2$ if $n$ is even.

\section{Deformations of double quiver algebras}

For each pair $\left(\mathcal{C}, \mathbb{Z}_{n}\right)$, we associate to it a quiver $\mathcal{Q}=\mathcal{Q}\left(\mathcal{C}, \mathbb{Z}_{n}\right)$ as follows. The set of vertices has a one-to-one correspondence with the group $\mathbb{Z}_{n}^{t}$. The set of arrows is

$$
\left\{a(x, i): x-c^{i} \rightarrow x \mid x \in Z_{n}^{t}, i \in \underline{t}\right\} .
$$

It is noticed that $e_{u} a(u, i)=a(u, i) e_{u-c^{i}}=a(u, i), \quad a(u, i)^{*} e_{u}=e_{u-c^{i}} a(u, i)^{*}=a(u, i)^{*}$ in the double quiver algebra $k \overline{\mathcal{Q}}$.

The following lemma is well known.

Lemma 2.1 ([2], Proposition 2.3) The path algebra $k \mathcal{Q}$ is a Hopf algebra where the comultiplication, counit, and antipode are given by the following

$$
\begin{aligned}
\Delta\left(e_{x}\right) & =\sum_{u+v=x} e_{u} \otimes e_{v}, \\
\Delta(a(x, i)) & =\sum_{u+v=x} q^{u_{i}} e_{u} \otimes a(v, i)+\sum_{u+v=x} a(u, i) \otimes e_{v}, \\
\varepsilon\left(e_{0}\right) & =1, \varepsilon\left(e_{x}\right)=0 \text { for all } x \neq 0, \varepsilon(a(x, i))=0, \\
S(a(x, i)) & =-q^{x_{i}-2} a\left(-x+c^{i}, i\right), S\left(e_{x}\right)=e_{-x}, \text { for all } x, i .
\end{aligned}
$$


We consider the quotient algebra $\Pi^{\mathcal{C}}=k \overline{\mathcal{Q}} / I$, where $I$ is the ideal generated by the following relations

$$
\begin{aligned}
& a(x, i) a(x, i)^{*}-a\left(x+c^{i}, i\right)^{*} a\left(x+c^{i}, i\right)-\frac{q^{x_{i}}-q^{-x_{i}}}{q-q^{-1}} e_{x}, \\
& a(x, j)^{*} a(x, i)-a\left(x-c^{j}, i\right) a\left(x-c^{i}, j\right)^{*}
\end{aligned}
$$

for all $x \in \mathbb{Z}_{n}^{t}, i, j \in \underline{t}$ with $i \neq j$.

The following fact is important to our aim.

Lemma 2.2 The algebra $\Pi^{\mathcal{C}}$ is a Hopf algebra with comultiplication, counit, and antipode given by

$$
\begin{aligned}
\Delta\left(e_{x}\right) & =\sum_{u+v=x} e_{u} \otimes e_{v}, \\
\Delta(a(x, i)) & =\sum_{u+v=x} q^{u_{i}} e_{u} \otimes a(v, i)+\sum_{u+v=x} a(u, i) \otimes e_{v}, \\
\Delta\left(a(x, i)^{*}\right) & =\sum_{u+v=x} e_{u} \otimes a(v, i)^{*}+\sum_{u+v=x} q^{-v_{i}} a(u, i)^{*} \otimes e_{v}, \\
\varepsilon\left(e_{0}\right) & =1, \quad \varepsilon\left(e_{x}\right)=0, \text { for all } x \neq 0, \\
\varepsilon(a(x, i)) & =0, \quad \varepsilon\left(a(x, i)^{*}\right)=0, \\
S(a(x, i)) & =-q^{x_{i}-2} a\left(-x+c^{i}, i\right), S\left(a(x, i)^{*}\right)=-q^{-x_{i}+2} a\left(-x+c^{i}, i\right)^{*}, \\
S\left(e_{x}\right) & =e_{-x}, \text { for all } x \in \mathbb{Z}_{n}^{t}, i \in \underline{t} .
\end{aligned}
$$

Proof. Let $\mathcal{Q}^{o p}$ be the reverse quiver of $\mathcal{Q}$. Then we have a path algebra $k \mathcal{Q}^{o p}$. By duality and Lemma 2.1, one can prove that $k \mathcal{Q}^{o p}$ is a Hopf algebra equipped with

$$
\begin{aligned}
\Delta\left(a(x, i)^{*}\right) & =\sum_{u+v=x} e_{u} \otimes a(v, i)^{*}+\sum_{u+v=x} q^{-v_{i}} a(u, i)^{*} \otimes e_{v}, \\
\varepsilon\left(e_{0}\right) & =1, \quad \varepsilon\left(e_{x}\right)=0, \text { for all } x \neq 0 \\
\varepsilon\left(a(x, i)^{*}\right) & =0, \\
S\left(a(x, i)^{*}\right) & =-q^{-x_{i}+2} a\left(-x+c^{i}, i\right)^{*}, \quad \text { for all } x, i . \\
S\left(e_{x}\right) & =e_{-x}, \text { for } x \in \mathbb{Z}_{n}^{t} .
\end{aligned}
$$

To see that $\Pi^{\mathcal{C}}$ is a Hopf algebra, it is sufficient to show that the given maps $\Delta$ and $\varepsilon$ keep 
the relations (2.1) and (2.2). Indeed, for the relation (2.1), we have

$$
\begin{aligned}
& \Delta(a(x, i)) \Delta\left(a(x, i)^{*}\right)-\Delta\left(a\left(x+c^{i}, i\right)^{*}\right) \Delta\left(a\left(x+c^{i}, i\right)\right) \\
= & \sum_{u+v=x} q^{u_{i}} e_{u} \otimes a(v, i) a(v, i)^{*}+\sum_{u+v=x} q^{-v_{i}} a(u, i) a(u, i)^{*} \otimes e_{v} \\
& -\sum_{u+v=x+c^{i}} q^{u_{i}} e_{u} \otimes a(v, i)^{*} a(v, i)-\sum_{u+v=x+c^{i}} q^{-v_{i}} a(u, i)^{*} a(u, i) \otimes e_{v} \\
= & \sum_{u+v=x} q^{u_{i}} e_{u} \otimes\left[a(v, i) a(v, i)^{*}-a\left(v+c^{i}, i\right)^{*} a\left(v+c^{i}, i\right)\right] \\
& +\sum_{u+v=x} q^{-v_{i}}\left[a(u, i) a(u, i)^{*}-a\left(u+c^{i}, i\right)^{*} a\left(u+c^{i}, i\right)\right] \otimes e_{v} \\
= & \sum_{u+v=x} q^{u_{i}} e_{u} \otimes \frac{q^{v_{i}}-q^{-v_{i}}}{q-q^{-1}} e_{v}+\sum_{u+v=x} q^{-v_{i}} \frac{q^{u_{i}}-q^{-u_{i}}}{q-q^{-1}} e_{u} \otimes e_{v} \\
= & \left(\frac{q^{x_{i}}-q^{-x_{i}}}{q-q^{-1}}\right) \sum_{u+v=x} e_{u} \otimes e_{v} .
\end{aligned}
$$

Hence $\Delta$ keeps the relation (2.1). For the relation (2.2), it is easy to see that

$$
\begin{aligned}
& \Delta\left(a(x, j)^{*}\right) \Delta(a(x, i))-\Delta\left(a\left(x-c^{j}, i\right)\right) \Delta\left(a\left(x-c^{i}, j\right)^{*}\right) \\
= & \sum_{u+v=x} q^{u_{i}} e_{u} \otimes\left[a(v, j)^{*} a(v, i)-a\left(v-c^{j}, i\right) a\left(v-c^{i}, j\right)^{*}\right] \\
& +\sum_{u+v=x} q^{-v_{i}}\left[a(u, j)^{*} a(u, i)-a\left(u-c^{j}, i\right) a\left(u-c^{i}, j\right)^{*}\right] \otimes e_{v} \\
= & 0
\end{aligned}
$$

The arguments for the counit $\varepsilon$ are similar. It remains to show that $S$ is an antipode. For example,

$$
\begin{aligned}
& S\left(a(x, i)^{*}\right) S(a(x, i))-S\left(a\left(x+c^{i}, i\right)\right) S\left(a\left(x+c^{i}, i\right)^{*}\right) \\
= & a\left(-x+c^{i}, i\right)^{*} a\left(-x+c^{i}, i\right)-a(-x, i) a(-x, i)^{*} \\
= & -\frac{q^{-x_{i}}-q^{x_{i}}}{q-q^{-1}} e_{-x}=\frac{q^{x_{i}}-q^{-x_{i}}}{q-q^{-1}} S\left(e_{x}\right) .
\end{aligned}
$$

For the relation (2.2) the argument for $S$ is similar (we note that $a_{i j}=a_{j i}$ ). Hence $S$ is the antipode of $\Pi^{\mathcal{C}}$. The lemma follows.

We denote the paths $a\left(x, i_{1}\right) a\left(x-c^{i_{1}}, i_{2}\right) \cdots a\left(x-c^{i_{1}}-\cdots-c^{i_{s-1}}, i_{s}\right)$ of $\overline{\mathcal{Q}}$ by $a\left(x, i_{1} i_{2} \cdots i_{s}\right)$. For brevity, $a(x, i) a\left(x-c^{i}, i\right) \cdots a\left(x-(s-1) c^{i}, i\right)$ is denoted by $a\left(x, i^{s}\right)$. Similarly, we use the notations $a\left(x, i_{1} i_{2} \cdots i_{s}\right)^{*}$ and $a\left(x, i^{s}\right)^{*}$. 
Lemma 2.3 We have the following formulae:

$$
\begin{aligned}
\Delta\left(a\left(x, i^{m}\right)\right) & =\sum_{\substack{u+v=x \\
s+t=m}}\left(\begin{array}{c}
m \\
s
\end{array}\right)_{q^{-2}} q^{t u_{i}} a\left(u, i^{s}\right) \otimes a\left(v, i^{t}\right) ; \\
\Delta\left(a\left(x, i^{m}\right)^{*}\right) & =\sum_{\substack{u+v=x \\
s+t=m}}\left(\begin{array}{c}
m \\
s
\end{array}\right)_{q^{2}} q^{-s v_{i}} a\left(u, i^{s}\right)^{*} \otimes a\left(v, i^{t}\right)^{*} .
\end{aligned}
$$

In particular, we have

$$
\begin{aligned}
\Delta\left(a\left(x, i^{\ell}\right)\right) & =\sum_{u+v=x} q^{\ell u_{i}} e_{u} \otimes a\left(v, i^{\ell}\right)+\sum_{u+v=x} a\left(u, i^{\ell}\right) \otimes e_{v} ; \\
\Delta\left(a\left(x, i^{\ell}\right)^{*}\right) & =\sum_{u+v=x} q^{-\ell v_{i}} a\left(u, i^{\ell}\right)^{*} \otimes e_{v}+\sum_{u+v=x} e_{u} \otimes a\left(v, i^{\ell}\right)^{*} .
\end{aligned}
$$

Proof. It is easy to see that the formula

$$
\left(\begin{array}{c}
m+1 \\
u
\end{array}\right)_{x}=\left(\begin{array}{c}
m \\
u
\end{array}\right)_{x}+x^{m-u+1}\left(\begin{array}{c}
m \\
u-1
\end{array}\right)_{x}
$$

holds. By this formula, the proof is completed by induction on $m$.

We set $\kappa=1-a_{i j}$ and for all $x \in \mathbb{Z}_{n}^{t}, i, j \in \underline{t}$ with $i \neq j$,

$$
\begin{aligned}
\omega_{i j}(x) & =\sum_{t=0}^{\kappa}(-1)^{t}\left[\begin{array}{c}
\kappa \\
t
\end{array}\right]_{q} a\left(x, i^{\kappa-t} j i^{t}\right) \\
\omega_{i j}(x)^{*} & =\sum_{t=0}^{\kappa}(-1)^{t}\left[\begin{array}{l}
\kappa \\
t
\end{array}\right]_{q} a\left(x, i^{\kappa-t} j i^{t}\right)^{*} .
\end{aligned}
$$

We have

Lemma 2.4 For all $x \in \mathbb{Z}_{n}^{t}, i, j \in \underline{t}$ with $i \neq j$,

$$
\begin{aligned}
\Delta\left(\omega_{i j}(x)\right) & =\sum_{u+v=x} q^{\kappa u_{i}+u_{j}} e_{u} \otimes \omega_{i j}(v)+\sum_{u+v=x} \omega_{i j}(u) \otimes e_{v} \\
\Delta\left(\omega_{i j}(x)^{*}\right) & =\sum_{u+v=x} e_{u} \otimes \omega_{i j}(v)^{*}+\sum_{u+v=x} q^{-\kappa v_{i}-v_{j}} \omega_{i j}(u)^{*} \otimes e_{v}
\end{aligned}
$$

Proof. The proof is considerably straightforward. We compute the formula $\Delta\left(\omega_{i j}(x)\right)$ where $a_{i j}=-1$. In this case,

$$
\omega_{i j}(x)=a\left(x, i^{2} j\right)-\left(q+q^{-1}\right) a(x, i j i)+a\left(x, j i^{2}\right) .
$$


We have

$$
\begin{aligned}
& \Delta\left(a\left(x, i^{2} j\right)\right)= \\
& \sum_{u+v=x} q^{2 u_{i}+u_{j}} e_{u} \otimes a\left(v, i^{2} j\right)+\sum_{u+v=x} q^{2 u_{i}} a(u, j) \otimes a\left(v, i^{2}\right) \\
& +\sum_{u+v=x}\left(q+q^{-1}\right) q^{u_{i}+u_{j}} a(u, i) \otimes a(v, i j) \\
& \quad+\sum_{u+v=x}\left(1+q^{-2}\right) q^{u_{i}} a(u, i j) \otimes a(v, i) \\
& \quad+\sum_{u+v=x} q^{u_{j}+2} a\left(u, i^{2}\right) \otimes a(v, j)+\sum_{u+v=x} a\left(u, i^{2} j\right) \otimes e_{v} .
\end{aligned}
$$

Similarly,

$$
\begin{aligned}
& \Delta\left(a\left(x, j i^{2}\right)\right)= \\
& \sum_{u+v=x} q^{2 u_{i}+u_{j}} e_{u} \otimes a\left(v, j i^{2}\right)+\sum_{u+v=x} q^{2 u_{i}+2} a(u, j) \otimes a\left(v, i^{2}\right) \\
& +\sum_{u+v=x}\left(1+q^{-2}\right) q^{u_{i}+u_{j}} a(u, i) \otimes a(v, j i) \\
& \quad+\sum_{u+v=x}\left(q+q^{-1}\right) q^{u_{i}} a(u, j i) \otimes a(v, i) \\
& +\sum_{u+v=x} q^{u_{j}} a\left(u, i^{2}\right) \otimes a(v, j)+\sum_{u+v=x} a\left(u, j i^{2}\right) \otimes e_{v},
\end{aligned}
$$

and

$$
\begin{aligned}
& \Delta(a(x, i j i))= \\
& \sum_{u+v=x} q^{2 u_{i}+u_{j}} e_{u} \otimes a(v, i j i)+\sum_{u+v=x} q^{u_{i}+u_{j}-1} a(u, i) \otimes a(v, j i) \\
& +\sum_{u+v=x} q^{2 u_{i}+1} a(u, j) \otimes a\left(v, i^{2}\right)+\sum_{u+v=x} q^{u_{i}-1} a(u, i j) \otimes a(v, i) \\
& +\sum_{u+v=x} q^{u_{i}+u_{j}} a(u, i) \otimes a(v, i j)+\sum_{u+v=x} q^{u_{j}+1} a\left(u, i^{2}\right) \otimes a(v, j) \\
& +\sum_{u+v=x} q^{u_{i}} a(u, j i) \otimes a(v, i)+\sum_{u+v=x} a(u, i j i) \otimes e_{v} .
\end{aligned}
$$

Therefore,

$$
\Delta\left(\omega_{i j}(x)\right)=\sum_{u+v=x} q^{2 u_{i}+u_{j}} e_{u} \otimes \omega_{i j}(v)+\sum_{u+v=x} \omega_{i j}(u) \otimes e_{v} .
$$

The cases when $a_{i j}=0$ and $\omega_{i j}(x)^{*}$ are similar.

Let $J$ be the ideal of $\Pi^{\mathcal{C}}$ generated by

$$
a\left(x, i^{\ell}\right), a\left(x, i^{\ell}\right)^{*}, \omega_{i j}(x), \omega_{i j}(x)^{*}
$$


for all $x \in \mathbb{Z}_{n}^{t}$ and $i, j \in \underline{t}$ with $i \neq j$. By the definition of the antipode, Lemmas 2.3 and 2.4, it is easy to see that $J$ is a Hopf ideal. We denote by $\mathfrak{u}_{q}^{\mathcal{C}}$ the quotient algebra $\Pi^{\mathcal{C}} / J$, which can be presented by generators and relations as follows. As an algebra, $\mathfrak{u}_{q}^{\mathcal{C}}$ is generated by $\left\{e_{x}, a(x, i), a(x, i)^{*} \mid x \in \mathbb{Z}_{n}^{t}, i \in \underline{t}\right\}$ with the following relations: for all $x \in \mathbb{Z}_{n}^{t}$ and $i, j \in \underline{t}$ with $i \neq j$,

$$
\begin{aligned}
& e_{x} e_{y}=\delta_{x y} e_{x}, e_{x} a(y, i)=a(y, i) e_{x-c^{i}}=\delta_{x, y} a(x, i), \\
& a(x, i)^{*} e_{y}=e_{y-c^{i}} a(x, i)^{*}=\delta_{x, y} a(x, i)^{*}, \\
& a(x, i) a(x, i)^{*}-a\left(x+c^{i}, i\right)^{*} a\left(x+c^{i}, i\right)=\frac{q^{x_{i}}-q^{-x_{i}}}{q-q^{-1}} e_{x}, \\
& a(x, j)^{*} a(x, i)=a\left(x-c^{j}, i\right) a\left(x-c^{i}, j\right)^{*} \\
& a\left(x, i^{\ell}\right)=0, a\left(x, i^{\ell}\right)^{*}=0 \\
& \omega_{i j}(x)=0, \omega_{i j}(x)^{*}=0 .
\end{aligned}
$$

We yield the result as follows.

Theorem $2.5 \mathfrak{u}_{q}^{\mathcal{C}}$ is a Hopf algebra, of which the comultiplication, counit, and antipode are defined by

$$
\begin{aligned}
\Delta\left(e_{x}\right) & =\sum_{u+v=x} e_{u} \otimes e_{v}, \\
\Delta(a(x, i)) & =\sum_{u+v=x} q^{u_{i}} e_{u} \otimes a(v, i)+\sum_{u+v=x} a(u, i) \otimes e_{v}, \\
\Delta\left(a(x, i)^{*}\right) & =\sum_{u+v=x} e_{u} \otimes a(v, i)^{*}+\sum_{u+v=x} q^{-v_{i}} a(u, i)^{*} \otimes e_{v}, \\
\varepsilon\left(e_{0}\right) & =1, \varepsilon\left(e_{x}\right)=0, \text { for all } x \neq 0, \\
\varepsilon(a(x, i)) & =0, \varepsilon\left(a(x, i)^{*}\right)=0, S(a(x, i))=-q^{x_{i}-2} a\left(-x+c^{i}, i\right), \\
S\left(a(x, i)^{*}\right) & =-q^{-x_{i}+2} a\left(-x+c^{i}, i\right)^{*}, \text { for all } x, i, \\
S\left(e_{x}\right) & =e_{-x}, \text { for all } x .
\end{aligned}
$$

\section{$3 \quad$ Isomorphisms between $\mathfrak{u}_{q}(\mathcal{C})$ and $\mathfrak{u}_{q}^{\mathcal{C}}$}

In this section, we keep all notations as before.

For $x=\left(x_{1}, \cdots, x_{t}\right)$, let $K_{x}=K_{1}^{x_{1}} \cdots K_{t}^{x_{t}}$ and $\epsilon_{x}=\frac{1}{n^{t}} \sum_{y \in \mathbb{Z}_{n}^{t}} q^{-x \cdot y} K_{y}$. By Lemma 1.1, it 
is easy to see that for any $x, y \in \mathbb{Z}_{n}^{t}$,

$$
\begin{aligned}
K_{y} \epsilon_{x} & =q^{x \cdot y} \frac{1}{n^{t}} \sum_{\beta \in \mathbb{Z}_{n}^{t}} q^{-x \cdot(\beta+y)} K_{\beta+y}=q^{x \cdot y} \epsilon_{x}, \\
\epsilon_{x} \epsilon_{y} & =\frac{1}{n^{t}} \sum_{\gamma \in \mathbb{Z}_{n}^{t}} q^{-x \cdot \gamma} K_{\gamma} \epsilon_{y}=\frac{1}{n^{t}} \sum_{\gamma \in \mathbb{Z}_{n}^{t}} q^{-(x-y) \cdot \gamma} e_{y}=\delta_{x, y} \epsilon_{x}, \\
\sum_{x \in \mathbb{Z}_{n}^{t}} \epsilon_{x} & =\sum_{x \in \mathbb{Z}_{n}^{t}} \frac{1}{n^{t}} \sum_{\beta \in \mathbb{Z}_{n}^{t}} q^{-x \cdot \beta} K_{\beta}=1+\frac{1}{n^{t}} \sum_{\substack{\beta \neq 0 \\
\beta \in \mathbb{Z}_{n}^{t}}}\left(\sum_{x \in \mathbb{Z}_{n}^{t}} q^{-x \cdot \beta}\right) K_{\beta}=1, \\
E_{i} \epsilon_{x} & =\epsilon_{x+c^{i}} E_{i}, F_{i} \epsilon_{x}=\epsilon_{x-c^{i}} F_{i}, \\
\Delta\left(\epsilon_{x}\right) & =\sum_{u+v=x} \epsilon_{u} \otimes \epsilon_{v}, S\left(\epsilon_{x}\right)=\epsilon_{-x} .
\end{aligned}
$$

We have constructed the Hopf algebra $\mathfrak{u}_{q}^{\mathcal{C}}$. The relationship between $\mathfrak{u}_{q}^{\mathcal{C}}$ and $\mathfrak{u}_{q}(\mathcal{C})$ is given as follows.

Theorem 3.1 The map $\tau: \mathfrak{u}_{q}^{\mathcal{C}} \rightarrow \mathfrak{u}_{q}(\mathcal{C})$ defined by

$$
\tau\left(e_{x}\right)=\epsilon_{x}, \tau(a(x, i))=\epsilon_{x} E_{i}, \tau\left(a(x, i)^{*}\right)=F_{i} \epsilon_{x}
$$

is a Hopf algebra isomorphism.

Proof. We prove the theorem in several steps.

Step 1: The map $\tau$ is well-defined. We should first verify that $\tau$ keeps the basic relations for path algebra. That is, $e_{x} e_{y}=\delta_{x y} e_{x}, e_{x} a(y, i)=a(y, i) e_{x-c^{i}}=\delta_{x y} a(y, i)$, and $a(x, i)^{*} e_{y}=$ $e_{y-c^{i}} a(x, i)^{*}=\delta_{x y} a(x, i)^{*}$. For the first one,

$$
\tau\left(e_{x}\right) \tau\left(e_{y}\right)=\epsilon_{x} \epsilon_{y}=\delta_{x, y} \epsilon_{x}=\tau\left(\delta_{x y} e_{x}\right)=\tau\left(e_{x} e_{y}\right)
$$

For the second one,

$$
\tau\left(e_{x}\right) \tau(a(y, i))=\epsilon_{x} \epsilon_{y} E_{i}=\delta_{x, y} \epsilon_{y} E_{i}=\tau\left(\delta_{x y} a(y, i)\right)
$$

The rest are similar.

It remains to show that $\tau$ keeps the relations (2.1)-(2.3). For example, for the relation 
$(2.1)$

$$
\begin{aligned}
& \tau(a(x, i)) \tau\left(a(x, i)^{*}\right)-\tau\left(a\left(x+c^{i}, i\right)^{*}\right) \tau\left(a\left(x+c^{i}, i\right)\right)-\frac{q^{x_{i}}-q^{-x_{i}}}{q-q^{-1}} \tau\left(e_{x}\right) \\
& =\epsilon_{x} E_{i} F_{i} \epsilon_{x}-\left(F_{i} \epsilon_{x+c^{i}}\right)\left(\epsilon_{x+c^{i}} E_{i}\right)-\frac{q^{x_{i}}-q^{-x_{i}}}{q-q^{-1}} \epsilon_{x} \\
& =\left(E_{i} F_{i}-F_{i} E_{i}\right) \epsilon_{x}-\frac{q^{x_{i}}-q^{-x_{i}}}{q-q^{-1}} \epsilon_{x} \\
& =\frac{K_{i}-K_{i}^{-1}}{q-q^{-1}} \epsilon_{x}-\frac{q^{x_{i}}-q^{-x_{i}}}{q-q^{-1}} \epsilon_{x} \\
& =\frac{q^{x_{i}}-q^{-x_{i}}}{q-q^{-1}} \epsilon_{x}-\frac{q^{x_{i}}-q^{-x_{i}}}{q-q^{-1}} \epsilon_{x}=0 .
\end{aligned}
$$

For the relation (2.2), it is similar. For the relation (2.3),

$$
\tau(a(x, i)) \cdots \tau\left(a\left(x-(\ell-1) c^{i}, i\right)=\left(\epsilon_{x} E_{i}\right) \cdots\left(\epsilon_{x-(\ell-1) c^{i}} E_{i}\right)=\epsilon_{x} E_{i}^{\ell}=0\right.
$$

and

$$
\begin{aligned}
& \sum_{t=0}^{\kappa}(-1)^{t}\left[\begin{array}{c}
\kappa \\
t
\end{array}\right]_{q} \tau(a(x, i)) \cdots \tau\left(a\left(x-(\kappa-t-1) c^{i}, i\right)\right. \\
& \quad \times \tau\left(a\left(x-(\kappa-t) c^{i}, j\right)\right) \\
& \quad \times \tau\left(a\left(x-(\kappa-t) c^{i}-c^{j}\right), i\right) \cdots \tau\left(a\left(x-(\kappa-1) c^{i}-c^{j}, i\right)\right) \\
& =\sum_{t=0}^{\kappa}(-1)^{t}\left[\begin{array}{c}
\kappa \\
t
\end{array}\right]_{q}\left(\epsilon_{x} E_{i}\right) \cdots\left(\epsilon_{x-(\kappa-t-1) c^{i}} E_{i}\right) \\
& \quad \times\left(\epsilon_{x-(\kappa-t-1) c^{i}-c^{j}} E_{i}\right)\left(\epsilon_{x-(\kappa-t) c^{i}} E_{j}\right) \cdots\left(\epsilon_{x-(\kappa-1) c^{i}-c^{j}} E_{i}\right) \\
& =\epsilon_{x}\left(\sum_{t=0}^{\kappa}(-1)^{t}\left[\begin{array}{c}
\kappa \\
t
\end{array}\right]_{q} E_{i}^{\kappa-t} E_{j} E_{i}^{t}\right)=0 .
\end{aligned}
$$

The rest relations in (2.3) are similar.

Step 2: Define an algebra map $\sigma: \mathfrak{u}_{q}(\mathcal{C}) \rightarrow \mathfrak{u}_{q}^{\mathcal{C}}$ by

$$
\sigma\left(K_{c}\right)=\sum_{x \in \mathbb{Z}_{n}^{t}} q^{c \cdot x} e_{x}, \sigma\left(E_{i}\right)=\sum_{x \in \mathbb{Z}_{n}^{t}} a(x, i), \sigma\left(F_{i}\right)=\sum_{x \in \mathbb{Z}_{n}^{t}} a(x, i)^{*}
$$

The aim is to show that $\sigma$ is the inverse of $\tau$. The map $\sigma$ is also well defined. Indeed, for the relation (0.1),

$$
\sigma\left(K_{i}\right)^{n}=\left(\sum_{x \in \mathbb{Z}_{n}^{t}} q^{x_{i}} e_{x}\right)^{n}=\sum_{x \in \mathbb{Z}_{n}^{t}} q^{n x_{i}} e_{x}=\sum_{x \in \mathbb{Z}_{n}^{t}} e_{x}=1
$$


since $q$ is an $n$-th root of unity. For the relation (0.2), we have

$$
\begin{aligned}
\sigma\left(K_{i}\right) \sigma\left(E_{j}\right) \sigma\left(K_{i}^{-1}\right) & =\left(\sum_{x \in \mathbb{Z}_{n}^{t}} q^{x_{i}} e_{x}\right)\left(\sum_{x \in \mathbb{Z}_{n}^{t}} a(x, j)\right)\left(\sum_{x \in \mathbb{Z}_{n}^{t}} q^{-x^{i}} e_{x}\right) \\
& =\sum_{x \in \mathbb{Z}_{n}^{t}} q^{x_{i}} e_{x} a(x, j) q^{-x_{i}+a_{i j}} e_{x-c^{j}} \\
& =q^{a_{i j}} \sum_{x \in \mathbb{Z}_{n}^{t}} a(x, j)=q^{a_{i j}} \sigma\left(E_{j}\right) .
\end{aligned}
$$

The another relation in $(0.2)$ is similar. For the relation $(0.3)$,

$$
\begin{aligned}
& \sigma\left(E_{i}\right) \sigma\left(F_{j}\right)-\sigma\left(F_{j}\right) \sigma\left(E_{i}\right) \\
= & \left(\sum_{x \in \mathbb{Z}_{n}^{t}} a(x, i)\right)\left(\sum_{x \in \mathbb{Z}_{n}^{t}} a(x, j)^{*}\right)-\left(\sum_{x \in \mathbb{Z}_{n}^{t}} a(x, j)^{*}\right)\left(\sum_{x \in \mathbb{Z}_{n}^{t}} a(x, i)\right) \\
= & \left\{\begin{array}{l}
\sum_{x \in \mathbb{Z}_{n}^{t}}\left[a(x, i) a(x, i)^{*}-a\left(x+c^{i}, i\right)^{*} a\left(x+c^{i}, i\right)\right], \quad \text { if } i=j ; \\
-\sum_{x \in \mathbb{Z}_{n}^{t}}\left[a(x, j)^{*} a(x, i)-a\left(x-c^{j}, i\right) a\left(x-c^{i}, j\right)^{*}\right], \quad \text { if } i \neq j
\end{array}\right. \\
= & \left\{\begin{array}{cc}
\sum_{x \in \mathbb{Z}_{n}^{t}} \frac{q^{x_{i}-q^{-x_{i}}}}{q-q^{-1}} e_{x}, & \text { if } i=j ; \\
0, & \text { if } i \neq j
\end{array}\right. \\
= & \sigma\left(\delta_{i j} \frac{K_{i}-K_{i}^{-1}}{q-q^{-1}}\right) .
\end{aligned}
$$

The relation (0.4) is due to the the first two relations of (2.3). As for the quantum Serre relations, if $a_{i j}=0$, we have

$$
\begin{aligned}
& \sigma\left(E_{i}\right) \sigma\left(E_{j}\right)-\sigma\left(E_{j}\right) \sigma\left(E_{i}\right) \\
= & \left(\sum_{x \in \mathbb{Z}_{n}^{t}} a(x, i)\right)\left(\sum_{x \in \mathbb{Z}_{n}^{t}} a(x, j)\right)-\left(\sum_{x \in \mathbb{Z}_{n}^{t}} a(x, j)\right)\left(\sum_{x \in \mathbb{Z}_{n}^{t}} a(x, i)\right) \\
= & \sum_{x \in \mathbb{Z}_{n}^{t}}[a(x, i j)-a(x, j i)]=0 ;
\end{aligned}
$$


if $a_{i j}=-1$, we have

$$
\begin{aligned}
& \sigma\left(E_{i}\right)^{2} \sigma\left(E_{j}\right)-\left(q+q^{-1}\right) \sigma\left(E_{i}\right) \sigma\left(E_{j}\right) \sigma\left(E_{i}\right)+\sigma\left(E_{j}\right) \sigma\left(E_{i}\right)^{2} \\
= & \left(\sum_{x \in \mathbb{Z}_{n}^{t}} a(x, i)\right)^{2}\left(\sum_{x \in \mathbb{Z}_{n}^{t}} a(x, j)\right)-\left(q+q^{-1}\right)\left(\sum_{x \in \mathbb{Z}_{n}^{t}} a(x, i)\right) \\
& \times\left(\sum_{x \in \mathbb{Z}_{n}^{t}} a(x, j)\right)\left(\sum_{x \in \mathbb{Z}_{n}^{t}} a(x, i)\right)+\left(\sum_{x \in \mathbb{Z}_{n}^{t}} a(x, j)\right)\left(\sum_{x \in \mathbb{Z}_{n}^{t}} a(x, i)\right)^{2} \\
= & \sum_{x \in \mathbb{Z}_{n}^{t}} \omega_{i j}(x)=0 .
\end{aligned}
$$

The arguments of the rest relations are similar.

By direct calculation, we have $\sigma \circ \tau=1$ and $\tau \circ \sigma=1$. Hence $\tau$ is an algebra isomorphism.

Step 3: $\tau$ is a Hopf algebra homomorphism. It is enough to verify that $\tau$ is also a coalgebra map since bialgebra homomorphisms are Hopf homomorphisms. It suffices to check it on the generators, but this is considerably direct. We check only one of them.

$$
\begin{aligned}
\Delta(\tau(a(i, x))) & =\Delta\left(\epsilon_{x} E_{i}\right)=\Delta\left(\epsilon_{x}\right) \Delta\left(E_{i}\right) \\
& =\left(\sum_{u+v=x} \epsilon_{u} \otimes \epsilon_{v}\right)\left(E_{i} \otimes 1+K_{i} \otimes E_{i}\right) \\
& =\sum_{u+v=x} \tau(a(u, i)) \otimes \tau\left(e_{v}\right)+\sum_{u+v=x} K_{i} \epsilon_{u} \otimes \tau(a(v, i)) \\
& =\sum_{u+v=x} \tau(a(u, i)) \otimes \tau\left(e_{v}\right)+\sum_{u+v=x} q^{u_{i}} \tau\left(e_{u}\right) \otimes \tau(a(v, i)) \\
& =(\tau \otimes \tau) \Delta(a(x, i)),
\end{aligned}
$$

The others are similar.

The proof is completed.

For the Cartan matrix $\mathcal{C}=\left(a_{i j}\right)_{t \times t}$, there is an associated Hopf algebra $\mathfrak{u}_{q}^{+}$generated by $K_{i}, E_{i}$ for $1 \leq i \leq t$, subjecting to the relations

$$
\begin{aligned}
& K_{i}^{n}=1, K_{i} K_{j}=K_{j} K_{i}, \\
& K_{i} E_{j} K_{i}^{-1}=q^{a_{i j}} E_{j} . \\
& \Delta\left(K_{i}\right)=K_{i} \otimes K_{i}, \\
& \Delta\left(E_{i}\right)=E_{i} \otimes 1+K_{i} \otimes E_{i} . \\
& \varepsilon\left(K_{i}\right)=1, \varepsilon\left(E_{i}\right)=0, \\
& S\left(K_{i}\right)=K_{i}^{-1}, S\left(E_{i}\right)=-K_{i}^{-1} E_{i} .
\end{aligned}
$$


Similarly, there is an associated Hopf algebra $\mathfrak{u}_{q}^{-}$generated by $K_{i}, F_{i}$ for $1 \leq i \leq t$, subjecting to the relations

$$
\begin{aligned}
& K_{i}^{n}=1, K_{i} K_{j}=K_{j} K_{i} ; \\
& K_{i} F_{j} K_{i}^{-1}=q^{-a_{i j}} F_{j} ; \\
& \Delta\left(K_{i}\right)=K_{i} \otimes K_{i}, \\
& \Delta\left(F_{i}\right)=F_{i} \otimes K_{i}^{-1}+1 \otimes F_{i}, \\
& \varepsilon\left(K_{i}\right)=1, \quad \varepsilon\left(F_{i}\right)=0, \\
& S\left(K_{i}\right)=K_{i}^{-1}, S\left(F_{i}\right)=-F_{i} K_{i} .
\end{aligned}
$$

By [11], there is a skew Hopf pairing $\mathfrak{u}_{q}^{+} \times \mathfrak{u}_{q}^{-} \rightarrow k$ and we have the Drinfeld double $\mathcal{D}\left(\mathfrak{u}_{q}\right)$ (see [11], Sect. 2). Let $I=\left\langle K_{i} \otimes 1-1 \otimes K_{i} \mid i \in \underline{t}\right\rangle$ be the ideal of $\mathcal{D}\left(\mathfrak{u}_{q}\right)$ generated by $K_{i} \otimes 1-1 \otimes K_{i}, i \in \underline{t}$. It is easy to see that $I$ is a Hopf ideal of $\mathcal{D}\left(\mathfrak{u}_{q}\right)$ and $\Pi^{\mathcal{C}} \cong \mathcal{D}\left(\mathfrak{u}_{q}\right) / I$ as Hopf algebras by Theorem 3.1. Furthermore, if $\underline{t}=\{1\}$, the relation (2.2) automatically vanishes and $\Pi^{\mathcal{C}}$ is just a deformation of preprojective algebra associated to the quiver $\mathcal{Q}$.

We have found an algebraic realization of the quantum group $\mathfrak{u}_{q}(\mathcal{C})$. This method is very intuitive. It is natural to expect that the presentation via a double quiver will help to study the representation theory, probably by consulting the theory of deformed preprojective algebras. This will be considered in the forthcoming papers.

\section{Some Remarks}

In the representation theory of finite dimensional algebras, finite dimensional basic algebras can always be constructed via quivers with admissible relations, according to Gabriel's Theorem. Recall that a path relation is called admissible if the length of the paths involved are at least two. We remark that our relation (2.1) is not admissible. Actually, the quantum groups $\mathfrak{u}_{q}(\mathcal{C})$ are not basic and hence there is no hope to present them via quivers with admissible relations. However it will be of interest to consider the Ext-quiver of the quantum groups and compare with the double quivers obtained.

For $\mathfrak{s l}_{2}$ the quiver obtained by the authors is the same as the quiver described in arXiv: math.RT/0410017, which appeared after the submission of our paper. The authors thank the referee for pointing out the reference.

\section{Acknowledgment}

The authors are grateful to the referee for his/her helpful comments and suggestions. 


\section{References}

[1] C. Cibils, A quiver quantum group, Comm. Math. Phys. 157 (1993) 459-477.

[2] C. Cibils, Half-quantum groups at roots of unity, path algebras, and representation type, Inter. Math. Res. Noticies 12 (1997) 541-553.

[3] W. Crawley-Boevey, M. P. Holland, Noncommutative deformations of Kleinian Singularities, Duke Math. J. 92 (1998) 605-635.

[4] Erdmann, K., Green, E. L., Snashall, N., Taillefer, R. Representation theory of the Drinfeld doubles of a family of Hopf algebras, preprint, arXiv: math.RT/0410017.

[5] J. A. Green, Hall algebras, hereditary algebras and quantum groups, Invent. Math. 120 (1995) 361-377.

[6] I. Gordon, Quantised function algebras at roots of unity and path algebras, J. Algebra 220 (1999) 381-395.

[7] G. Lusztig, Modular representations and quantum groups, Contemp. Math. 82 (1989) 59-77.

[8] G. Lusztig, Finite dimensional Hopf algebras arising from quantized universal enveloping algebras, Journal of the AMS 3 (1990) 257-296.

[9] G. Lusztig, Quantum groups at roots of 1, Geom. Dedicata 35 (1990) 89-113.

[10] C. M. Ringel, Hall algebras and quantum groups, Invent. Math. 101 (1990) 583-592.

[11] J. Xiao, Drinfeld double and Ringel-Green theory of Hall Algebras, J. Algebra 190 (1997) 100-144.

[12] Shilin Yang, Quantum groups and deformations of preprojective algebras, J. Algebra, 279 (2004), 3-21. 